\title{
Time Delays in Acute Myocardial Infarction - the Gender Perspective
}

\section{Imre Benedek}

University of Medicine and Pharmacy, Tîrgu Mureș, Romania

Acute myocardial infarction (AMI) is still responsible for a high number of deaths in developed countries, despite of many efforts to improve the healthcare strategy addressed to this devastating disease. Different regional models of dedicated networks have been proposed in an attempt to reduce the mortality associated with AMI in the acute phase. Some of these networks have been proved to represent real life-saving therapies, as the implementation of primary revascularization in ST-elevation myocardial infarction (STEMI) within a well-organized system led to a spectacular reduction in STEMI-related mortality. ${ }^{1-3}$

In an attempt to make the STEMI networks highly efficient, the new guidelines of the European Society of Cardiology have established specific targets for STEMI-related time intervals. ${ }^{4} \mathrm{~A}$ significant part of the logistic and organizational efforts are addressed to reduce the time from the first medical contact (FMC) to arrival in a pPCI center or from the FMC to revascularization.

However, a major factor that is usually underestimated in the STEMI networking strategies is the significant delay from symptoms onset to presentation in the emergency department. As this critical time is a major component of the total ischemic time, this delay leads to prolongation of the total ischemic time, which is directly associated with worse outcomes in both the acute and post-infarction phase.

The time from symptoms onset to presentation to the emergency room depends on the patient's awareness regarding angina symptoms, the degree of perception of the imminent risk of having a myocardial infarction, and, implicitly, on the degree of patient education. A significant tendency to delay presentation in case of angina symptoms is encountered in low-income countries, as a result of poorer economic status, societal factors, and lower level of health education. A study published in 2007 by Ting et al. reported a time from symptoms onset to FMC of 99 minutes in case of direct presentation to a hospital facility with catheterization laboratory in North America. ${ }^{5}$ In contrast, populations from low-income countries have significantly larger time delays, reaching even 200 minutes from symptoms onset to presentation in the emergency department in Romania.

A study published in this number of JCE by Anghel et al., reported significantly longer delays from symptoms to presentation in the emergency room in case of a female population from North East Romania suffering an AMI, in comparison to men. ${ }^{6}$ According to this study, fewer women than men presented to the hospital in the first 12 hours after angina onset. Interestingly, the authors also demonstrated that in women from this geographical area, coronary events began more frequently in the morning with atypical symptoms. It should be noted, however, that in this study, the delay from symptoms onset to diagnosis was not caused by the fact that the angina symptoms were non-specific, but rather by the ignorance of women in relation to angina symptoms characteristics.

It is well-known that in the first decades of life, women are more protected against cardiovascular diseases by their hormonal balance. However, this changes at the menopause, when the hormonal protection disappears, and women become exposed to heart attacks in a similar manner to men. The fact that opposite to high-income 
countries, in low-income countries infarct-related symptoms are more likely to be ignored by women shows that there are significant disparities in the gender-related perspective of different organizational healthcare models.

This study underlines to need for proper organizational measures addressed to the society in order to educate the population on how to recognize the symptoms of an acute heart attack.

\section{CONFLICT OF INTEREST}

Nothing to declare.

\section{REFERENCES}

1. Rhudy JP, Bakitas MA, Hyrkäs K, et al. Effectiveness of regionalized systems for stroke and myocardial infarction. Brain Behav. 2015;5:e00398. doi:10.1002/brb3.398.

2. Smith FG, Brogan RA, Alabas $\mathrm{O}$, et al. Comparative care and outcomes for acute coronary syndromes in Central and Eastern European Transitional countries: A review of the literature. Eur Heart J Acute Cardiovasc Care. 2015;4:537-554. doi: $10.1177 / 2048872614551545$.

3. Benedek I, Gyongyosi M, Benedek T. A prospective regional registry of ST-elevation myocardial infarction in Central Romania: impact of the Stent for Life Initiative recommendations on patient outcomes. Am Heart J. 2013;166:457-465. doi: 10.1016/j.ahj.2013.03.033

4. Ibanez B, James S, Agewall S, et al. 2017 ESC Guidelines for the management of acute myocardial infarction in patients presenting with ST-segment elevation: The Task Force for the management of acute myocardial infarction in patients presenting with ST-segment elevation of the European Society of Cardiology (ESC). Euro Heart J. 2018;39:119-177. doi: https://doi.org/10.1093/eurheartj/ehx393.

5. Ting HH, Rihal CS, Gersh BJ, et al. Regional systems of care to optimize timeliness of reperfusion therapy for STelevation myocardial infarction: the Mayo Clinic STEMI Protocol. Circulation 2007;116:729-736. doi: 10.1161/ CIRCULATIONAHA.107.699934

6. Larisa Anghel, Cristina Prisacariu, Cătălina Arsenescu Georgescu. Gender-Related Particularities in Acute Myocardial Infarction - a Study on a Patient Cohort from North East Romania. Journal of Cardiovascular Emergencies. 2018;4:95100. doi: $10.2478 /$ jce-2018-0011. 